\title{
The First Workers of the Ant Camponotus obscuripes Are a Different Allometric Morph with Relatively Long Antennae to Communicate with Other Larger Colony Members
}

\author{
Saori Watanabe, ${ }^{1}$ Mamoru Terayama, ${ }^{2}$ Ryota Kawauchiya, ${ }^{3}$ Natsuki Ogusu, ${ }^{1}$ \\ Yusuke Fujita, ${ }^{1}$ Syunta Mikami, ${ }^{1}$ Yuuka Murakami, ${ }^{4}$ and Eisuke Hasegawa ${ }^{1}$ \\ ${ }^{1}$ Laboratory of Animal Ecology, Department of Ecology and Systematics, Graduate School of Agriculture, Hokkaido University, \\ Sapporo 060-8589, Japan \\ ${ }^{2}$ Laboratory of Applied Entomology, Department of Agricultural and Environmental Biology, The University of Tokyo, \\ Tokyo 113-8657, Japan \\ ${ }^{3}$ Institute of Entomology, Department of Ecology and Systematics, Graduate School of Agriculture, Hokkaido University, \\ Sapporo 060-8589, Japan \\ ${ }^{4}$ Department of Neuropharmacology, Graduate School of Medicine, Hokkaido University, Sapporo 060-8638, Japan
}

Correspondence should be addressed to Eisuke Hasegawa; ehase@res.agr.hokudai.ac.jp

Received 30 March 2017; Revised 19 May 2017; Accepted 28 May 2017; Published 22 June 2017

Academic Editor: Abraham Hefetz

Copyright (C) 2017 Saori Watanabe et al. This is an open access article distributed under the Creative Commons Attribution License, which permits unrestricted use, distribution, and reproduction in any medium, provided the original work is properly cited.

\begin{abstract}
The first workers produced by an ant queen with a claustral founding mode are much smaller than the workers after the second generation and are thus called "nanitics." These nanitics shoulder the initial fate of the colony and thus may be different morphometric morph from the other workers in mature colony to optimize the survival of their own colony. We report here that, in the ant Camponotus obscuripes Mayr, the allometric rules of the nanitics are different from those of other workers in mature colonies, suggesting that the nanitics constitute an independent caste as with soldiers or queens in other species. In addition, the antennae of the nanitics show the minimum absolute length-difference with the mother queen compared to the other traits measured. This result suggests that this small size difference enables $C$. obscuripes nanitics to communicate with the other members of the colony. Our results indicate that polymorphic societies affect the growth rules of workers.
\end{abstract}

\section{Introduction}

Many ant species show the claustral nest-founding mode, in which a dealated queen produces a few 1st workers using only the reserved nutrition in her body [1-3]. Eclosed 1st workers are much smaller than the workers after the 2nd generation and are thus called "nanitics" [2]. The nanitics are special workers that shoulder the fate of the colony. Since the amount of resources that can be invested into the production of the nanitics is limited to the stored nutrients in the queen's body, a trade-off between size and numbers is expected to occur among the nanitics produced by a queen [4]. In fact, this trade-off has been detected in the nanitics of the monogynous ant Camponotus japonicus Mayr, 1866 [5]. A previous study showed that there is a negative correlation between the number and the size of the nanitics when a queen's investment into them was controlled statistically [5]. Therefore, the nanitics are assumed to be under strict constraints in terms of their development to maintain the optimal relationship between size and number. This suggests that the nanitics may have different growth rules relating morphological traits to body size compared with other workers in mature colonies.

From the analysis of the slope and the intercept of this equation, we can determine the growth rules of the traits. In some cases, the allometric rule of a trait changes with body size, namely, a morphological polymorphism. For example, males of a stag beetle, Prosopocoilus inclinatus (Motschulsky, 1857), show a dimorphism in the mandible size to the body 
size [6]. The male mandible of stag beetles is a special organ used to fight with other males, and the two morphs adopt different reproductive strategies $[7,8]$. Thus, the growth rules are affected by the ecology of the focal organism.

In ants, worker polymorphisms are classified into five types, that is, monomorphism, monophasic allometry, diphasic allometry, triphasic allometry, and complete dimorphism [9]. A species showing distinctive polymorphisms has more than one worker-morph, each of which has a different allometric rule for some traits, for example, the small normal workers and the soldiers with a huge head in species of the ant genus Pheidole [complete dimorphism [10]]. In this distinct polymorphism, each caste has a different allometric rule for some trait (e.g., the head of workers in Atta spp.). In this type, the allometric line has one (or more) inflection point(s), meaning that there is more than one morph, each of which has a different allometric rule $[9,11]$. Another type is monophasic allometry, in which the size of workers varies considerably within a colony, but the allometric rule is represented by a simple linear regression, with Camponotus obscuripes Mayr 1879 belonging to this type [12].

In a monophasic allometric ant, Solenopsis invicta Buren, 1972, allometric rules have been shown to differ depending on the colony size or the social form (monogyny or polygyny) [13]. In S. invicta, the allometric equation of the ratio of the antennae/body size to the body length has a negative slope [13]. In our interpretation, this result suggests that the antennae of S. invicta are under a constraint in terms of their absolute length, likely because the ants communicate with other colony members by physical contacts with the antennae, and thus, communication may occur only between workers with antennae of a similar length.

We generalized two hypotheses to be tested from this consideration; (1) antennae are the smallest divergent trait between small and large individuals in an ant species, and (2) nanitics may have different allometric rules for several traits (especially for antennae) comparing with workers in mature colonies, meaning that nanitics are a distinct morphological caste as with soldiers in the complete dimorphic species.

In this study, we tested these hypotheses using a monophasic allometric ant, C. obscuripes. We compared the allometric rules for several traits of the nanitics and workers in mature colonies. In addition, we checked the degree of the difference in absolute length for examined traits between the nanitics and their mother queens. Based on the results, we tested the above hypotheses and will discuss how the social lives of ants affect the morphological growth rules of workers belonging to different generations.

\section{Materials and Methods}

2.1. Study Organisms. We examined a monophasic allometric ant, Camponotus obscuripes. Eighteen incipient colonies were obtained (see below). Each individual was separated into the head, thorax, right-side legs, and abdomen. These body parts were fixed to a glass slide using an adhesive agent and were dried at $60^{\circ} \mathrm{C}$ for 3 days before the measurements. All the measured traits are exoskeleton parts, and thus, the drying did not shrink the sizes of the traits.

The allometric rules of the workers in mature colonies were examined using a sample from a mature colony from Niigata Pref., Japan. Thirty-three workers that covered the full size range of the colony were prepared as described in the previous paragraph. All the individual body parts were photographed with a scale bar $(10 \mathrm{~mm})$ using a digital camera (WRAYCAM-NF300, WRAYMER, Osaka, Japan) attached to a binocular microscope (SZH, OLYMPUS, Tokyo, Japan).

In autumn 2016, eighteen incipient colonies (including a queen and a few nanitics) of C. obscuripes were bought from an online insect shop (the Ant-Room; http://www.antroom .jp/about.php). All the queens were collected in the summer of 2016 in Hachioji, Tokyo, Japan.

2.2. Morphological Measurements. We measured several morphological traits of all the individuals (see Figure 1; head width (HW), head length (HL), thorax length (TL), pronotum length $(\mathrm{PN})$, length of right forefemur (FF), foretibia (FT), midfemur (MF), midtibia (MT), hindfemur (HF), hindtibia (HT), width of posterior end of 1st segment of abdomen (AW), and lengths of the left antennal scape (AS) and the antennal funiculus (AF)) to $0.001 \mathrm{~mm}$ using a freeware (ImageJ, ver. 1.45) on the digital photographs. For the mature colony, we selected 33 workers that covered the full size range of the sample. For the incipient colonies, we measured 18 queens and a total of 78 nanitics ( 2 to 7 nanitics per queen).

First, we conducted a principal component analysis of the workers in the mature colony to determine the index of body size. As the antennae are focal objectives, we removed AS and AF from this analysis. Relationships of growth among organs are represented by the allometric rules between two traits [14]. In an allometric analysis, a trait is selected as the index of body size (for the selection method, see [15], pp 203-205), and the regression of other traits on the body size is calculated using the log-transformed data. The trait that is the most isometric with the principal component 1 is used as the index of body size (see [15], pp 203-205). AW was selected as the index of body size. Then, we calculated allometric equations for the remaining traits based on AW for the nanitics, the workers in the mature colonies, and the queens using log-transformed data. Then, linear regression equations were calculated for each class. The slopes and the intercepts of the two allometric equations for each trait pair were compared within the 3 classes using ANCOVA.

Second, we compared the absolute sizes of each trait between the queen and her nanitics as (the worker's length)/(the queen's length) to determine which trait shows the lowest size difference between the queens and the nanitics. Using the 76 data points for each trait, we compared each pair of traits for significant differences between the two traits using the Wilcoxon matched pairs test. The significance level was corrected using the Bonferroni correction for multiple comparisons. In addition, we calculated the same ratios for the workers in the mature colony between the largest and smallest workers (in AW). 


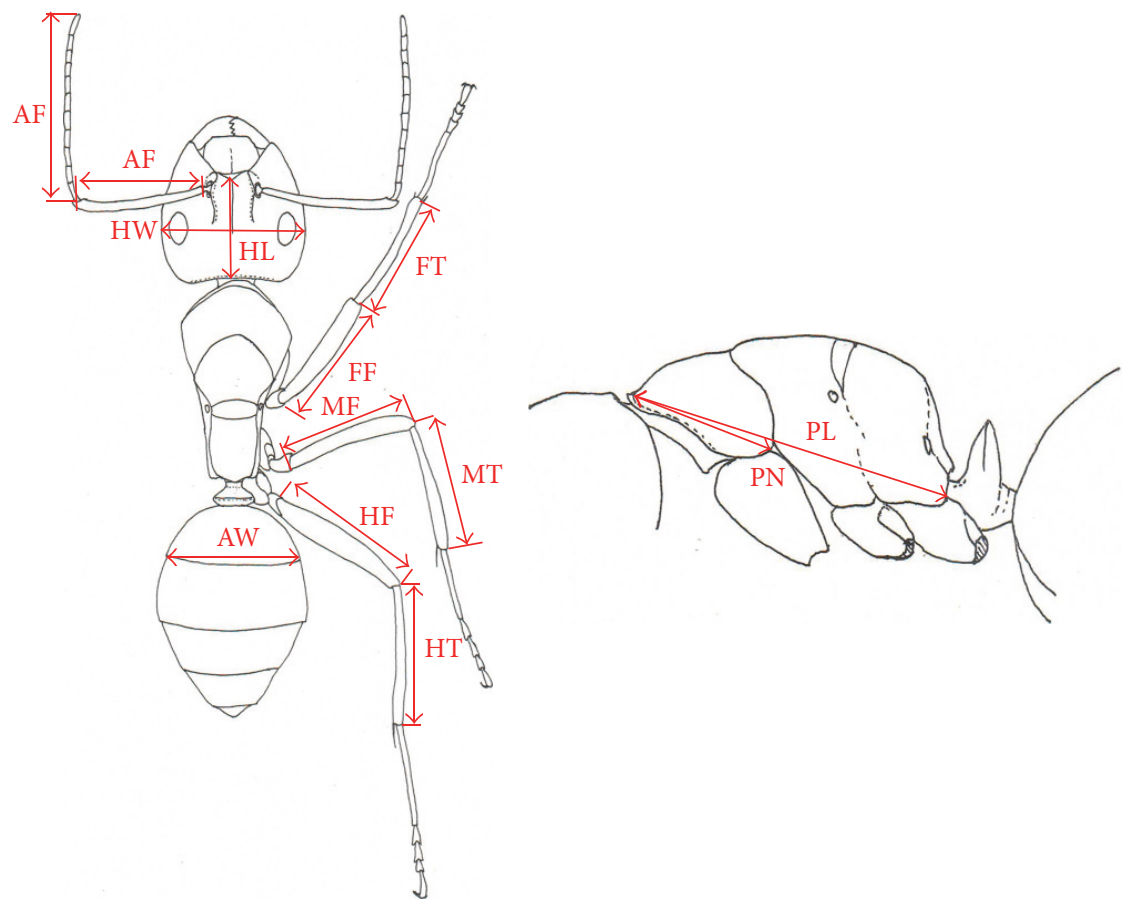

AF: antennal funiculus
AS: antennal scape
HW: head width
HL: head length
TL: thorax length
PN: pronotum length

AF: antennal funiculus HL: head length PN: pronotum length

$$
\begin{aligned}
& \text { FF: right forefemur } \\
& \text { FT: right foretibia } \\
& \text { MF: right midfemur } \\
& \text { MT: right midtibia } \\
& \text { HF: right hindfemur } \\
& \text { HT: right hind tibia }
\end{aligned}
$$

FIGURE 1: The traits measured.

\section{Results}

First, there is no significant correlation between the sizes of the same trait of the queens and her nanitics for all the measured traits (Pearson's correlation coefficient; for all the traits, $p>0.05$ ), meaning that we can treat each nanitic's data as an independent data point because these results showed that there is no phenotypic correlation between the mother queen and her nanitics. Probably, the expected genetic correlation has been covered by differences in nutritional condition among queens because sizes of nanitics have shown to be affected by the initial amount of resources stored in their queen [5]. Thus, we combined all the data for the incipient colonies for the statistical analyses.

Figure 2 shows the allometric relationships for each trait in each class (nanitics, workers in a mature colony, and queens). In several traits, there is a difference in the allometric slopes between the nanitics and the other workers (i.e., HW, $\mathrm{HL}, \mathrm{TL}, \mathrm{FF}, \mathrm{FT}$, and MF); the differences are significant after the Bonferroni correction for multiple comparisons (see Figure 2). But note that, for AF, there is no difference in the slopes among the three classes. For the workers in the mature colonies, only the slope of HW is significantly steeper than 1.0 , that is, the monophasic allometry in them. The slopes of MT, HF, and HT do not differ between the nanitics and the other workers. In conclusion, the nanitic is a distinct caste that follows different growth rules from those of the other workers for several traits.

Figure 3 shows the proportions of a dwarf trait to the same trait of their mother queen (Figure 3(a)) and those between the largest and smallest workers in the mature colony (Figure 3(b)). In the former, AS and AF are the traits that show the first and second smallest differences in absolute length compared to the queens. These differences are still significant after the Bonferroni correction for multiple comparisons (Figure 3(a)). As a result, the two parts of antennae (AF and AS) have the lowest differences in length compared to the queen. Although we could not conduct a statistical test, this trend is the same for the workers in the mature colony (Figure 3(b)).

\section{Discussion}

Our first finding is that the nanitics of C. obscuripes make up a distinct morphological caste that has different allometric rules for several traits from those of the other workers in the mature colony. The existence of nanitics in claustral founding ants has been thought to be a cause of their success in the ecosystem [3]. Because of the limited amount of resources needed to produce nanitics, a trade-off between size and 

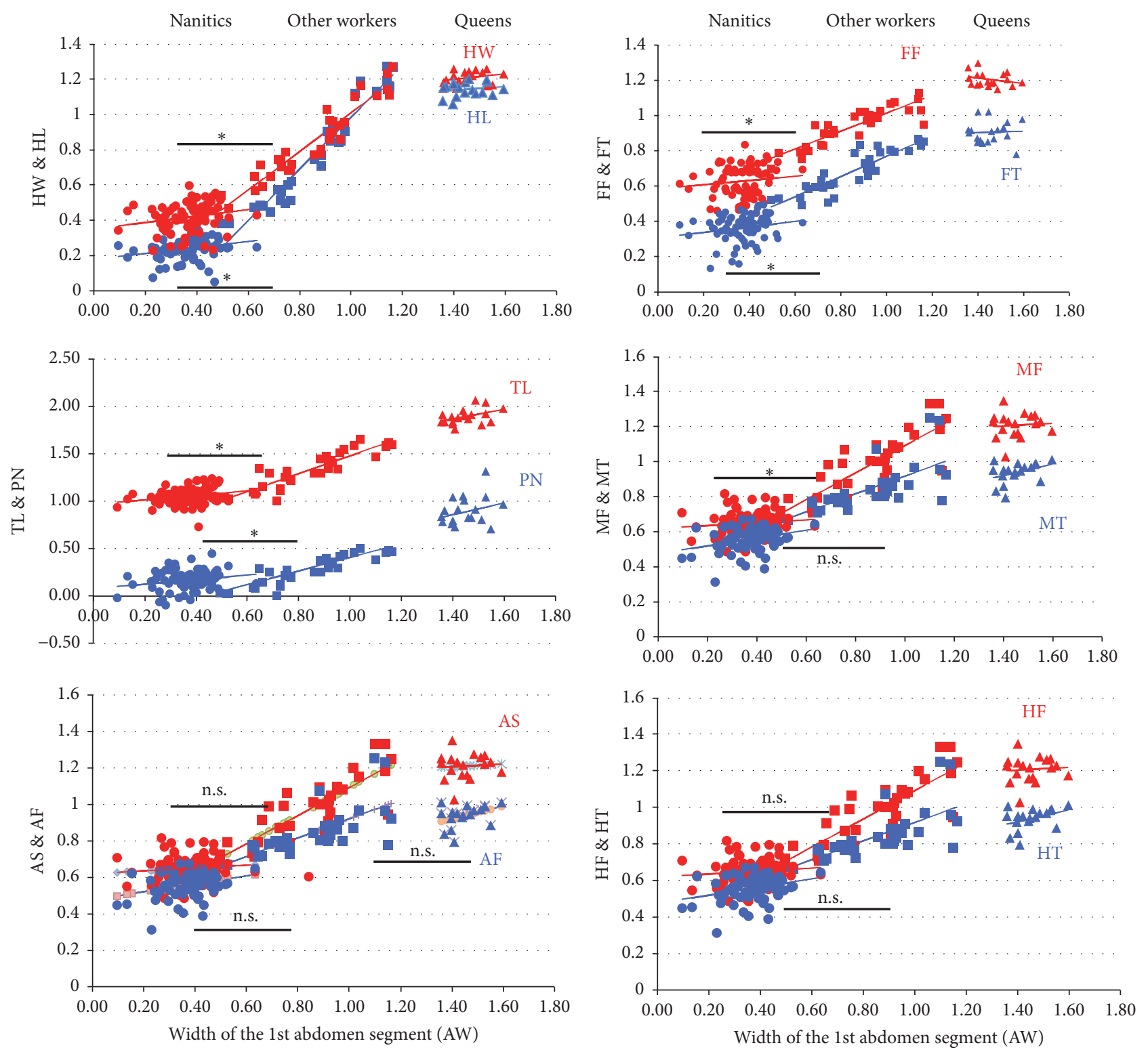

FIGURE 2: Allometric pattern in each trait for each class (nanitics (left circle), other workers (central squares), and queens (right triangles)). Significant differences among the classes (the compared class is shown with horizontal black bars) were tested by ANCOVA. The stars (*) represent a statistically significant difference in the slopes after Bonferroni correction for multiple comparisons among the classes (the significance level is $p=0.0042$ in this case). The fact that the queens are a distinct caste is apparent; the queen class was removed from the calculations excluding the analysis for AF, in which the allometric slope for the queens is not different from those of the other two classes.

number is expected [4], and a previous study has supported this hypothesis [5]. Because nanitics shoulder the initial fate of the colony, their body shape should be strongly constrained to the optimal growth rule to maximize colony survival. Thus, it is not surprising that the nanitics have distinct allometric rules from those of other workers. Our results demonstrate this, and we conclude that nanitics of $C$. obscuripes constitute a distinct caste from the other workers, like soldiers in species with the complete dimorphism.

A distinctive allometric pattern among the 3 classes (nanitics, other workers, and queens) has led us to a conclusion that $C$. obscuripes is a species showing the diphasic allometry rather than the monophasic allometry as believed previously [9]. The workers in a mature colony have a simple linear regression slope in each trait (Figure 2) suggesting they show the monophasic allometry, but the nanitics show significantly different slopes for several traits (i.e., HW, HL, TL, PN, FF, FT, and MF) from the workers in the mature colony (Figure 2). These results suggest that other ant species showing the monophasic allometry may be substantially diphasic as with C. obscuripes. Examinations of allometric rules in nanitics are needed to confirm this hypothesis.

The diphasic allometry typically found in HW, that is, the allometric slope, is moderate in the nanitics and the queens 


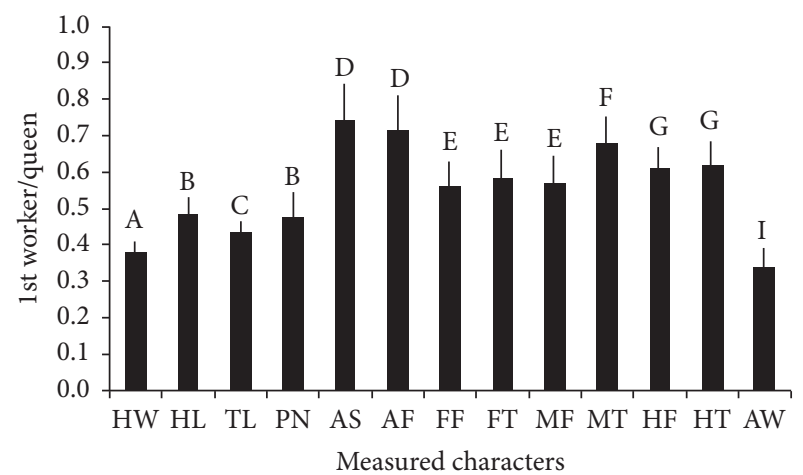

(a)

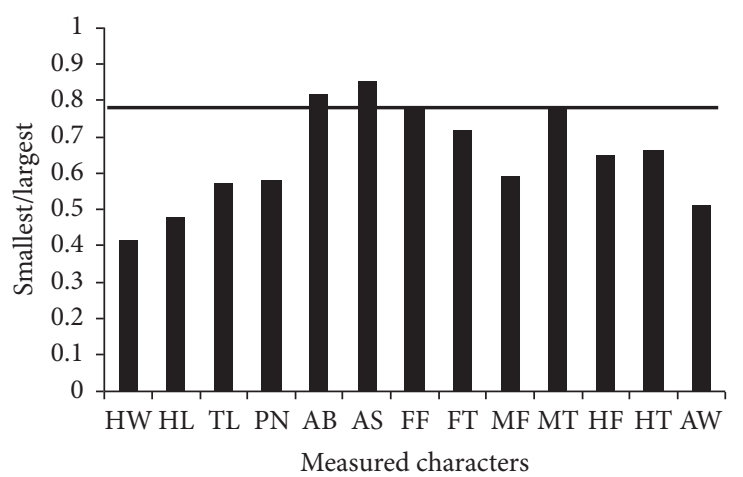

(b)

FIGURE 3: The ratios of a dwarf's trait length to her mother queen's length of the same trait. (a) The ratio of dwarf to queen traits. The different letters on the whiskers indicate statistically significant differences after Bonferroni correction for multiple comparisons. (b) The ratio of the smallest workers to the largest ones in the mature colony. AS and AF show the first and second smallest differences in these lengths. The black horizontal bar in (b) shows the value of the trait that shows the third smallest difference (FF).

but is steep in the other workers (see Figure 2). This pattern is found in several other traits. For example, TL, PN, FF, FT, and MF show similar patterns with HW. It is noteworthy that these traits are thought to support directly the weight of the head. A previous study has shown that workers of ants have relatively large pronotum in which there are many neck muscles that support head weight [16]. The allometric slope of HW (1.472) is significantly steeper than 1 (ANCOVA, $t=11.095, \mathrm{df}=32, p=1.679 e-12$ ), indicating that HW increases disproportionately with body size. As HW becomes large, the head weight will increase to the third power of the increase in HW. Thus, supportive traits of the head weight would show a similar allometric pattern as that of HW. In male stag beetles with exaggerated mandibles as weapons, supportive traits of the weapons (head or prothorax) are also enlarged $[17,18]$.

It is unknown why the worker's head disproportionally enlarges with body size in C. obscuripes. The enlarged head of the large workers in C. obscuripes may function as a food crusher as in soldiers of Pheidole spp. [10]. Nevertheless, an enlarged head would need robust supportive traits to be fully functional. Therefore, several such traits (TL, PN, FF, FT, and MF) may show a similar allometric growth pattern as that of HW.

The hind leg does not show the above pattern and instead seems to be monomorphic with AW. Hind legs may be used mainly to support the weight of the abdomen and thus increase constantly with abdomen size irrespective of the worker size. This hypothesis seems to be supported by the observed allometric patterns found in HF and HT.

The second finding is the smallest difference in the absolute length of antennae between large and small individuals. Figure 3(a) shows the proportions of a nanitic's trait size to her mother's. The AS and AF of the nanitics are significantly closer to those of the queen compared with the other traits after a Bonferroni correction for multiple comparisons (Figure 2). Ants communicate with nest mates using their antennae. In particular, the antennal funiculus seems to be important because it contains many sensory receptors [19]. If there is a large difference in the AF between individuals, efficient communications may be difficult.

This constraint comes from sociality. As colony members must communicate with nest mates to maintain colony function, the length of the antennae cannot differ substantially among the nest members. Solitary insects would be free from this constraint and, thus, the observed feature of the antennae may be restricted in social insects. However, we cannot test this hypothesis using ants because there is no ant species without sociality, excluding the social parasites (the antennae of a social parasite would become of a similar length to that of the host species due to the requirement for communicating with host individuals). However, in social wasps belonging to the Polistinae, the 1st workers are smaller than those after the second generation [20]. If social Polistinae wasps showed the same trend in antennal length as that of $C$. obscuripes, we could test the size constraint hypothesis by comparing the difference in antennal length to the body size between solitary and social wasps. The smallest difference in antennal length between large and small individuals would be a trait that has evolved after acquiring sociality. This hypothesis is worth being tested in future studies. In conclusion, the acquisition of sociality differently affects the growth rules of different castes in a colony to optimize colony efficiency for survival.

\section{Disclosure}

The English in this document has been checked by a professional editor who is a native speaker of English. The certificate is available by Eisuke Hasegawa. The funders had no role in study design, data collection and analysis, decision to publish, or preparation of the manuscript.

\section{Conflicts of Interest}

The authors declare no financial conflicts of interest. 


\section{Acknowledgments}

This work was partly supported by Grants-in-Aid from the Ministry of Education, Culture, Sports, Science and Technology of Japan to Eisuke Hasegawa (nos. 26440228 and 15H04420).

\section{References}

[1] E. O. Wilson, The Insect Societies, Harvard University Press, Cambridge, Mass, USA, 1971.

[2] B. Hölldobler and E. O. Wilson, The Ants, Harvard University Press, Harvard, Mass, USA, 1990.

[3] C. Peeters and F. Ito, "Wingless and dwarf workers underlie the ecological success of ants (Hymenoptera: Formicidae)," Myrmecological News, vol. 21, pp. 117-130, 2015.

[4] G. F. Oster and E. O. Wilson, Caste and Ecology in The Social Insect, Princeton University Press, Princeton, NJ, USA, 1978.

[5] E. Hasegawa and S. Imai, "A trade-off between number and size within the first workers of the ant Camponotus japonicus," Journal of Ethology, vol. 30, no. 1, pp. 201-204, 2012.

[6] Y. Okada, H. Fujisawa, Y. Kimura, and E. Hasegawa, "Morphdependent form of asymmetry in mandibles of the stag beetle Prosopocoilus inclinatus (Coleoptera: Lucanidae)," Ecological Entomology, vol. 33, no. 5, pp. 684-689, 2008.

[7] Y. Okada and E. Hasegawa, "Size-dependent precopulatory behavior as mate-securing tactic in the Japanese stag beetle, Prosopocoilus inclinatus (Coleoptera; Lucanidae)," Journal of Ethology, vol. 23, no. 2, pp. 99-102, 2005.

[8] A. Inoue and E. Hasegawa, "Effect of morph types, body size and prior residence on food-site holding by males of the maledimorphic stag beetle Prosopocoilus inclinatus (Coleoptera: Lucanidae)," Journal of Ethology, vol. 31, no. 1, pp. 55-60, 2013.

[9] E. O. Wilson, "The origin and evolution of polymorphism in ants," The Quarterly Review of Biology, vol. 28, no. 2, pp. 136156, 1953.

[10] E. O. Wilson, Pheidole in the New World-A Dominant, Hyper Divers Ant Genus, Harvard University Press, Cambridge, Mass, USA, 2003.

[11] J. L. Tomkins, J. S. Kotiaho, and N. R. Lebas, "Matters of scale: positive allometry and the evolution of male dimorphisms," The American Naturalist, vol. 165, no. 3, pp. 389-402, 2005.

[12] M. Terayama, "The ant genus Camponotus Mayr (Hymenoptera: Japan) in Japan," Memories of Myrmecological Society of Japan, vol. 1, pp. 25-48, 1999.

[13] M. B. Araujo and W. R. Tschinkel, "Worker allometry in relation to colony size and social form in the fire ant solenopsis invicta," Journal of Insect Science, vol. 10, no. 94, pp. 1-10, 2010.

[14] S. J. Gould, "Allometry and size in ontogeny and phylogeny," Biological Reviews, vol. 41, no. 4, pp. 387-640, 1966.

[15] P. H. Harvey and M. D. Pagel, The Comparative Method in Evolutionary Biology, Oxford University Press, New York, NY, USA, 1998.

[16] R. A. Keller, C. Peeters, and P. Beldade, "Evolution of thorax architecture in ant castes highlights trade-off between flight and ground behaviors," eLife, vol. 2014, no. 3, Article ID e01539, 2014.

[17] J. Goyens, J. Dirckx, and P. Aerts, "Stag Beetle Battle Behavior and its Associated Anatomical Adaptations," Journal of Insect Behavior, vol. 28, no. 3, article no. 1, pp. 227-244, 2015.
[18] J. Ito, Y. Ohkubo, and E. Hasegawa, "Morph-specific weaponcorrelated traits in a male dimophic stag beetle (Coleoptera: Lucanidae)," Annals of Entomological Society of America.

[19] M. Ozaki, A. Wada-Katsumata, K. Fujikawa et al., "Ant nestmate and non-nestmate discrimination by a chemosensory sensillum," Science, vol. 309, no. 5732, pp. 311-314, 2005.

[20] S. Miyano S, "Amount of flesh food influences the number, larval duration, and body size of first brood workers, in a Japanese paper wasp, Polistes chinensis antennalis (Hymenoptera: Vespidae)," Entomological Science, vol. 1, no. 4, pp. 545-549, 1998. 

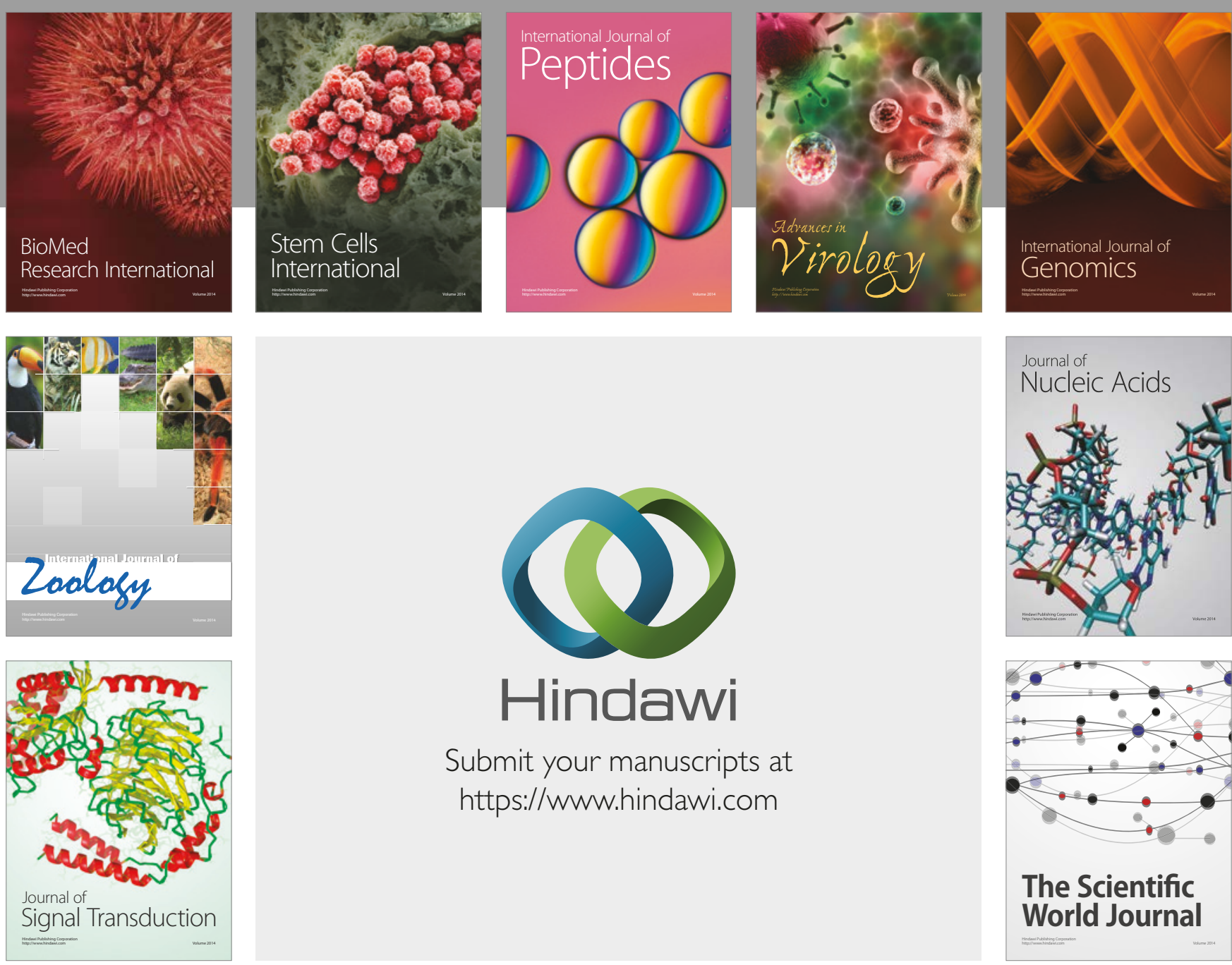

Submit your manuscripts at

https://www.hindawi.com
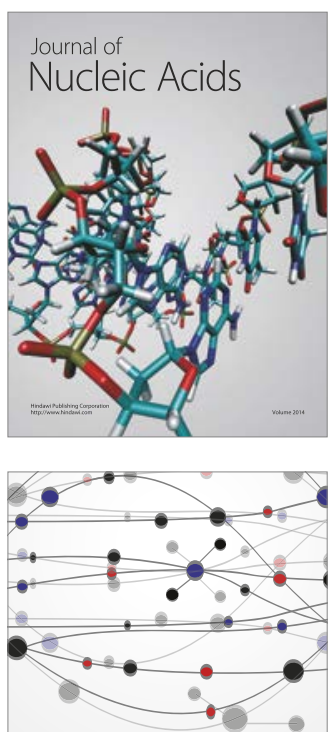

The Scientific World Journal

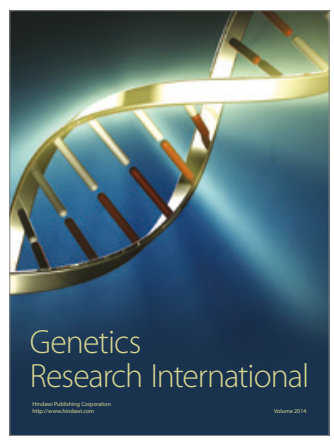

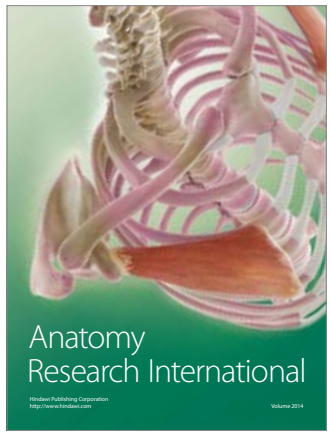

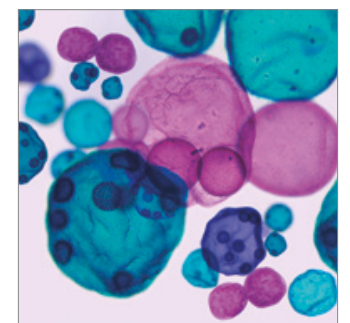

International Journal of Microbiology
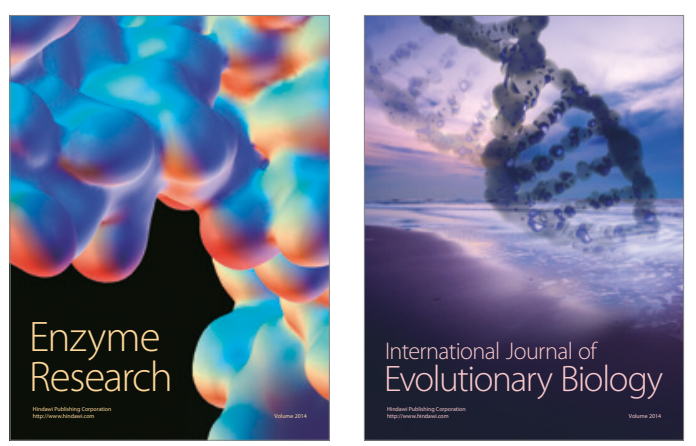
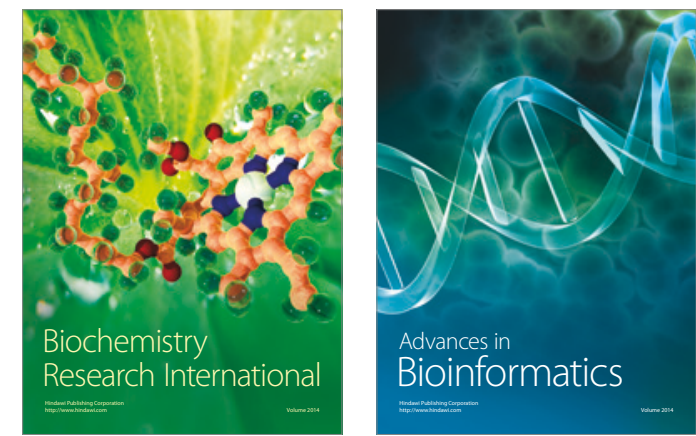

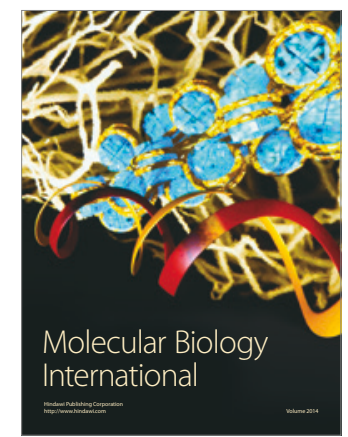

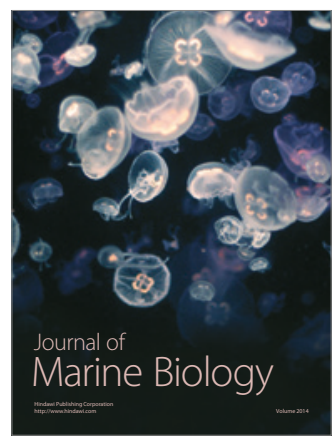

